\title{
Mortality Monitoring in the Netherlands
}

\author{
Liselotte van Asten*1, Marit de Lange ${ }^{1}$, Carel Harmsen², Kees van den Wijngaard ${ }^{1}$, Mirjam \\ Kretzschmar $^{1,3}$ and Wim van der Hoek ${ }^{1}$
}

${ }^{1}$ National Institute for Public Health and the Environment, Centre for Infectious Disease Control Netherlands, Bilthoven, Netherlands; ${ }^{2}$ Statistics Netherlands, Department of Demographic and socio-economic Statistics, The Hague, Netherlands; ${ }^{3}$ University Medical Center Utrecht, Julius Center for Health Sciences and Primary Care, Utrecht, Netherlands

\section{Objective}

Weekly numbers of deaths are monitored to increase the capacity to deal with unusual (disease) events such as pandemic influenza, other infections and non-infectious incidents. The monitoring information can potentially be used to track and estimate the impact of an outbreak on all cause mortality.

\section{Introduction}

The mortality monitoring system was initiated in august 2009 during the influenza $\mathrm{A}(\mathrm{H} 1 \mathrm{~N} 1)$ pandemic. It is a collaboration between the Centre for Infectious Disease Control (CIb) and Statistics Netherlands. The system monitors nation-wide reported number of deaths (population: 16.8 million) from all causes, as cause of death information is not available real-time. Data is received from Statistics Netherlands by weekly emails.

\section{Methods}

Once a week the number of reported deaths is checked for excess above expected levels at 3 different time-lags: deaths reported within 1,2 and 3 weeks after date of death (covering a median $43 \%, 96 \%$ and $98 \%$ of all deaths respectively). A weekly email bulletin reporting the findings is sent to the Infectious Disease Early Warning Unit (at CIb). Any known concurrent and possibly related events are also reported. When excess deaths coincide with hot temperatures the bulletin is sent to the Heat Plan Team (like CIb, within the National Institute for Public Health and the Environment (RIVM)). Data are also sent to EuroMOMO which monitors excess mortality at a European level. For the Dutch system, baselines and prediction limits are calculated using a 5 year historical period (updated each July). A serfling-type algorithm is used to produce baselines which includes cyclical seasonal trends (models based on historical data in which weeks with extreme underreporting have been removed. Also periods with high excess mortality in winter and summer were removed so as not to influence the baseline with previous outbreaks).

\section{Results}

In the $2012 / 2013$ winter season there is a long 18 -week period (week 50-16, but not week 52) with excess mortality (deaths reported within two weeks), largely coinciding with the exceptionally long influenza period. During this timeperiod influenza A \& B circulated but also RSV circulation and a cold snap occurred - factors that partially coincided with each other. The excess amounted to approximately $6,000+$ deaths during those weeks, a considerable increase above the average excess during influenza periods. During a 1-week heat wave in July a slight increase was seen in mortality in 65-74 year olds.

\section{Conclusions}

Comparison of different levels of prediction intervals could guide the choice of the optimal alert level for each level of reported deaths. The timelier (but less complete) data of deaths reported within 1 week showed a signal 5 weeks earlier, long before the influenza season, indicating that a higher prediction limit might be considered (based on $4 \mathrm{SD}$ instead of $3 \mathrm{SD}$, removing most of the very early and late alerts that were not reflected in the more complete but later data). The weekly bulletin will eventually be made publicly available through a website.

\section{Keywords}

Mortality monitoring; Public Health Surveillance; Influenza

*Liselotte van Asten

E-mail: Liselotte.van.asten@rivm.nl 